\title{
Negative dielectrophoresis spectroscopy for rare analyte quantification in biological samples
}

\author{
Syed Abdul Mannan Kirmani \\ Fleming Dackson Gudagunti \\ Logeeshan Velmanickam \\ Dharmakeerthi Nawarathna \\ Ivan T. Lima, Jr.
}




\title{
Negative dielectrophoresis spectroscopy for rare analyte quantification in biological samples
}

\author{
Syed Abdul Mannan Kirmani,, ${ }^{\mathrm{a} b}$ Fleming Dackson Gudagunti, ${ }^{\mathrm{a}}$ Logeeshan Velmanickam, ${ }^{\mathrm{a}}$ \\ Dharmakeerthi Nawarathna, ${ }^{a}$ and Ivan T. Lima Jr. ${ }^{a, *}$ \\ ${ }^{a}$ North Dakota State University, Electrical and Computer Engineering Department, Fargo, North Dakota, United States \\ ${ }^{\mathrm{b}}$ COMSATS Institute of Information Technology, Center for Advanced Studies in Telecommunication, Islamabad, Pakistan
}

\begin{abstract}
We propose the use of negative dielectrophoresis (DEP) spectroscopy as a technique to improve the detection limit of rare analytes in biological samples. We observe a significant dependence of the negative DEP force on functionalized polystyrene beads at the edges of interdigitated electrodes with respect to the frequency of the electric field. We measured this velocity of repulsion for $0 \%$ and $0.8 \%$ conjugation of avidin with biotin functionalized polystyrene beads with our automated software through real-time image processing that monitors the Rayleigh scattering from the beads. A significant difference in the velocity of the beads was observed in the presence of as little as 80 molecules of avidin per biotin functionalized bead. This technology can be applied in the detection and quantification of rare analytes that can be useful in the diagnosis and the treatment of diseases, such as cancer and myocardial infarction, with the use of polystyrene beads functionalized with antibodies for the target biomarkers. @ 2017 Society of Photo-Optical Instrumentation Engineers (SPIE) [DOI: 10.1117/1.JBO.22.3.037006]
\end{abstract}

Keywords: biomedical optics; biophotonics; sensors; real-time imaging; Rayleigh scattering.

Paper 160813R received Nov. 29, 2016; accepted for publication Mar. 14, 2017; published online Mar. 30, 2017.

\section{Introduction}

Early diagnosis is vital for successful treatment of many lifethreatening medical conditions. ${ }^{1,2}$ Accurate diagnosis of diseases and the subsequent prognosis by a physician involves a number of laboratory tests. Many tests involve detecting and quantifying rare biomolecules in the biological samples using biosensors. ${ }^{3}$ A biosensor essentially consists of a sensitive biological element, which reacts selectively with the target analyte and a transducer or detector with associated signal processing and output display. ${ }^{4}$ The biosensors use a biomolecule, such as an enzyme, protein, antibody, nucleic acid, etc., as an active detection agent. ${ }^{3-5}$ Broadly, biosensors are categorized into either catalytic or affinity biosensors. ${ }^{4}$ The catalytic biosensors employ an active biomolecule, such as an enzyme, that reacts specifically with some rare analytes. ${ }^{4}$ The affinity biosensors exploit the specific interaction of an analyte (ligand) and a biological receptor. ${ }^{4}$ Immunosensor is essentially an affinity biosensor that exploits antibody-antigen interactions. ${ }^{6-8}$ Antibodies are glycoprotein molecules called immunoglobulins that are produced by plasma cells as an immune response induced by an antigen. ${ }^{4}$ When incorporated into a biosensor, the antibody is either adsorbed or covalent bonded to a solid surface, such as a polystyrene well plate, as in cases of enzyme-linked immunosorbent assay (ELISA) or to polystyrene beads as in latex agglutination assay (LA). ${ }^{3,5,7,9}$ Then, the biological sample containing the target analytes is either pipetted, flowed, or mixed with the immunoassay. ${ }^{5,10}$ Any antigen molecules present in the sample react readily with the antibody in the assay, and agglutination occurs. ${ }^{11,12}$ There are many different techniques to detect and quantify the agglutination in LA and ELISA, such as dielectrophoresis (DEP) or electrochemical impedance spectroscopy. ${ }^{13-18}$ In DEP, various target biological particles, such as antigens, antibodies, cells, or DNA, can be detected, isolated, concentrated, or purified. ${ }^{4,19-21}$ In an earlier publication from our research group, ${ }^{5}$ we reported a dielectrophoretic label-free immunoassay for rare analyte quantification in biological samples with the capability to detect and quantify about 850 avidin molecules attached to biotin functionalized polystyrene beads. We are now reporting an improved method in which automated quantitative measurement of DEP was achieved through image processing and the DEP spectrum curves were obtained for two different concentrations of avidin-biotin conjugates over a range of frequencies. These standard curves can be utilized to detect the presence of avidin molecules in a solution. This method can be extended to the detection of rare biomarkers with the use of polystyrene beads functionalized with the appropriate antibodies for those biomarkers. Our method, which is based on the negative DEP spectroscopy, has the potential to be used as a primary point-of-care diagnostic technique because it makes use of reusable microscopic pearl-shaped interdigitated (PID) electrode array and it is capable of detecting a very low concentration of target biomolecules. The quantification of rare analytes very important for detecting various stages of tumors in oral and prostate cancer. ${ }^{1,2,5}$ Similarly, early detection of raised troponin level in blood can help in diagnosis and treatment of myocardial infarction. ${ }^{22-24} \mathrm{We}$ can adapt our technique for other rare analytes by replacing biotin with another binding antibody for the respective target analytes.

\section{Theory}

Dielectrophoresis (DEP) is the translational movement of electrically neutral polarizable particles in a nonuniform electric field. ${ }^{4,25}$ It is exploited in biotechnology for its remarkable capability of selectively isolating, concentrating, or purifying 
target particles present within a complex mixture by either attracting or repelling these target particles from the electrodes. ${ }^{19,26,27}$ The translational forces of attraction or repulsion arise from the interaction of the dipole moment of the particles with the nonuniform electric field. ${ }^{28}$

The time-averaged DEP force on a spherical particle of radius $r$ is given as ${ }^{4,5,19,25}$

$$
\left\langle F_{\mathrm{DEP}}\right\rangle=2 \pi \varepsilon_{o} \varepsilon_{m} r^{3} \operatorname{Re}\left[K_{\mathrm{CM}}(\omega)\right] \nabla E^{2},
$$

where $\varepsilon_{o}$ is the permittivity of the free space, $\varepsilon_{m}$ is the relative permittivity of the medium, $\nabla E$ is the electric field gradient, and $\operatorname{Re}\left[K_{\mathrm{CM}}(\omega)\right]$ is the real part of the Clausius-Mossotti factor [that is, effective polarizability (per unit volume) of the particle], which is given as a $^{4,5,19,25}$

$$
K_{\mathrm{CM}}(\omega) \equiv \frac{\varepsilon_{p}^{*}-\varepsilon_{m}^{*}}{\varepsilon_{p}^{*}+2 \varepsilon_{m}^{*}},
$$

where $\varepsilon_{p}^{*}$ is the complex permittivity of the particle and $\varepsilon_{m}^{*}$ is the complex permittivity of the medium. The complex permittivity is related to the conductivity $\sigma$ and the angular frequency $\omega$ of the applied alternating current field through the following equation: ${ }^{4,5,19,25}$

$$
\varepsilon^{*} \equiv \varepsilon-j \frac{\sigma}{\omega}
$$

Depending on the relative values of the permittivity of the particle and the medium, the value of $K_{\mathrm{CM}}(\omega)$ varies from -0.5 to 1 . If $K_{\mathrm{CM}}(\omega)$ is positive, then particles are attracted to regions of high electric field intensity, an effect termed positive DEP. Similarly, if $K_{\mathrm{CM}}(\omega)$ is negative, then the particles are repelled from the regions of high electric field intensity, an effect termed negative DEP. Since $K_{\mathrm{CM}}(\omega)$ is frequency dependent, both positive and negative DEP effects can be observed by changing the frequency of the applied electric field. The crossover frequency $f_{x o}$ is defined as the frequency at which the force changes from positive to negative DEP. It is given as $4,5,19,25,29$

$f_{x o}=\frac{1}{2 \pi} \sqrt{-\frac{\left(\sigma_{p}-\sigma_{m}\right)\left(\sigma_{p}+2 \sigma_{m}\right)}{\left(\varepsilon_{p}-\varepsilon_{m}\right)\left(\varepsilon_{p}+2 \varepsilon_{m}\right)}}$,

where $\sigma_{p, m}$ and $\varepsilon_{p, m}$ are the conductivity and the relative permittivity, respectively, of the particle and of the medium. The value of $f_{x o}$ depends on the conductivity of the particle $\sigma_{p}$ at low frequencies $(<1 \mathrm{MHz})$ and on the permittivity of the particle at higher frequencies. The total conductivity $\sigma_{p}$ of a spherical dielectric particle is given by the sum of its bulk $\sigma_{\text {bulk }}$ and surface conductivity $K_{s}^{4,5,19,25}$

$\sigma_{p}=\sigma_{\text {bulk }}+\frac{2 K_{s}}{r}$

Since $\sigma_{\text {bulk }}$ of the very small particles is negligible, the $\sigma_{p}$ is mainly dependent on $K_{s}$. Depending on the size and conjugation of the polystyrene beads, the value of $K_{s}$ varies in order of magnitude and provides a means to isolate, concentrate, or separate different types of target bioparticles. ${ }^{30-33}$

\section{Negative DEP Spectroscopy Application}

We have developed a Microsoft Foundation Classes (MFC) application in Visual C++ for Microsoft Windows operating system to obtain the negative DEP spectrum of dielectric particles dissolved in a conductive solution. Our application can capture live video and time-lapse images from any universal serial bus (USB) video class standard compliant microscope camera and perform real-time image processing to efficiently measure the effect of DEP force on dielectric particles and obtain the negative DEP spectrum curve. Our application captures and displays live video from a microscope camera with a frame rate of 25 frames per second. The frame rate can be increased or decreased to a value within hardware constraints. The application then converts the captured frames into grayscale and performs real-time image processing to obtain useful information.

The dialog window in Fig. 1 shows the measurement window, where the live video feed is displayed after being converted to grayscale. The application has the complete pixel information, including the color, of the captured video frames in computer memory and can perform various real-time image processing tasks to obtain useful information. Since we are interested in efficiently analyzing the effect of the DEP force on the suspended particles in the conducting medium as a function of the frequency of the electric field, we look for regions of interest in the video where strong a DEP effect is visible. These regions of interest are the areas where the beads accumulate in the cases of positive or negative DEP. These regions of interest are marked with rectangles, squares, or parallelograms. The image area within these regions of interest is now processed and recorded for efficiently characterizing DEP force and calculating the DEP spectrum curve. We can set a desired interval to capture timelapse images for further processing and record data values during the experiment. The minimum time interval to capture an image or record a new data value is $40 \mathrm{~ms}$, which is determined by the frame rate of the video stream and processing velocity of the hardware. We can also specify different time intervals for positive and negative DEP analysis. Since the suspended particles take some time to accumulate along the edges of the electrode when positive DEP is observed, additional time is needed to analyze this effect. However, in the case of negative DEP, the accumulated beads along the edges of the electrodes are readily repulsed from the electrodes when the electric signal is applied. Therefore, negative DEP requires much less time for examination. We also control the function generator by software to automatically scan through a set of electrical frequencies over the specified intervals of time. We can specify the start frequency, the frequency step, the stop frequency, the peak-to-peak voltage, and the time interval for the positive DEP and the negative DEP frequencies.

For our experiment, the start frequency was $500 \mathrm{kHz}$, the frequency step was $300 \mathrm{kHz}$, and the stop frequency was $2 \mathrm{MHz}$, which produces a negative DEP spectrum with six measurements. The peak-to-peak voltage value was $10 \mathrm{~V}$. The time interval for positive DEP was $30 \mathrm{~s}$ and the time interval for negative DEP was $4 \mathrm{~s}$ per frequency measurement. To measure the negative DEP spectrum, the experiment starts with a certain frequency $f_{p}$ that induces strong positive DEP effect for a specified time interval to concentrate the polystyrene beads at the edges of the electrodes. When the time interval of the positive DEP elapses, the frequency of the function generator is automatically changed to the first frequency $f_{n, 1}$ that induces negative DEP effect for the specified time interval. As the time interval for 


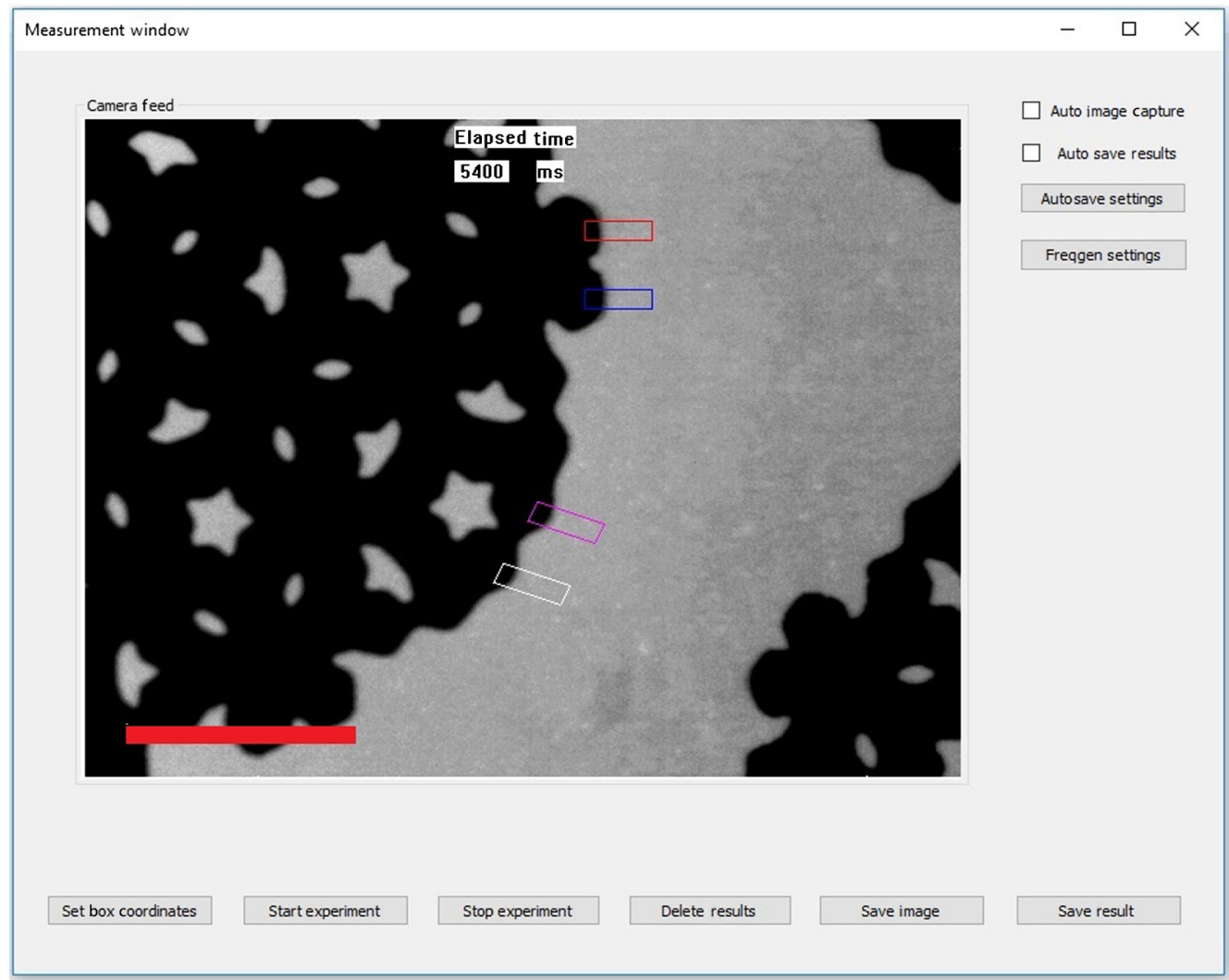

Fig. 1 Microsoft Windows application for DEP spectroscopy. The electrodes are visible in the darker regions in the picture. The red scale bar indicates $100 \mu \mathrm{m}$. The hollow rectangles depict the regions of interest for measurements and analysis.

frequency $f_{n, 1}$ is elapsed, the frequency is switched back to the frequency $f_{p}$ to transport the beads to the region with high electric field at the edges of the electrodes. Then, the next negative DEP frequency $f_{n, 2}$, which corresponds to the previous negative DEP frequency $f_{n, 1}$ incremented by the frequency step size, is applied. This cycle goes on until the frequency $f_{n, N}$ reaches or exceeds the stop frequency. All frequency switching by the function generator is done automatically by our application. Our application is designed for the Tektronix AFG series function generators. The function generator was connected to the computer via USB port. We modified an Interchangeable Virtual Instruments Foundation compliant device driver from LabWindows ${ }^{\mathrm{TM}} / \mathrm{CVI}$ environment to Microsoft Visual $\mathrm{C}++$. This modified device driver along with TekVISA (Virtual Instrument Software Architecture) connectivity software was used to access and control the function generator from Microsoft Visual C++ program.

\section{Experimental Results}

We validated our software by performing experiments with a PID electrode array that was designed and fabricated by our group. The electrode was designed and drawn to scale in AutoCAD, validated in the COMSOL Multiphysics software package, and fabricated on commercially available glass wafer using photolithography, metal sputtering, and lift-off procedures using 1000- $\AA$-thick gold film. Our electrode is capable of generating the maximum electric field of $1.8 \times 10^{4} \mathrm{~V} / \mathrm{m}$, which is sufficient for polarizing the polystyrene beads for microscopic observation of DEP. It was reported earlier in Ref. 5 that average electric field and electric field gradients do not vary with frequency in our electrode. This ensured that the polystyrene beads are subjected to the same electric field gradients in all frequencies and their DEP behavior is only dependent on the value of $\operatorname{Re}\left[K_{\mathrm{CM}}(\omega)\right]$, which is significantly dependent on the number of analyte molecules attached to the bead surface. We studied the variation of DEP force on biotin functionalized polystyrene beads with and without a small number of avidin molecules per bead at various frequencies. The biotin functionalized polystyrene beads with $0.74-\mu \mathrm{m}$ diameter $(10,000$ biotin molecules on each bead surface) were purchased from Spherotech Inc. These biotinylated beads were conjugated with fluorescently labeled avidin molecules $(1 \mathrm{mg} / \mathrm{mL})$ purchased from Vector Labs Inc. through the manufacturer suggested procedure. To achieve $100 \%$ avidin-biotin conjugation (i.e., 10,000 biotin molecules on the surface of polystyrene beads attached to 10,000 avidin molecules), we incubated $3 \mu \mathrm{L}$ of avidin solution and $10 \mu \mathrm{L}$ biotin functionalized polystyrene beads for $30 \mathrm{~min}$ at room temperature. We then centrifuged the solution at $5000 \mathrm{rpm}$ for $12 \mathrm{~min}$ to separate functionalized beads from the solution. After centrifugation, the supernatant was removed and $390 \mu \mathrm{L}$ of $0.01 \times$ diluted phosphate-buffer saline (PBS) with conductivity $0.01 \mathrm{~S} / \mathrm{m}$ was added. The $10 \mu \mathrm{L}$ of the prepared conjugated bead solution was pipetted onto the commercially available glass slide for microscopic observation during the experiment. Similarly, $0.8 \%$ avidin-biotin conjugated solutions were prepared by diluting the avidin solution appropriately and keeping other parameters (incubation time, temperature, and centrifuge velocity) constant. 
The $0 \%$ solution contained only biotin functionalized polystyrene beads. We conducted experiments for $0 \%$ and $0.8 \%$ avidinbiotin conjugated solutions.

For the experiment, we mounted a clean PID electrode array on OMFL600 low-power microscope and employed a side illumination technique for observations. We used a custom-made green light-emitting diode (LED) lamp illuminating from an angle of incidence of $45 \mathrm{deg}$. This angle of incidence reduces the amount of light collected by the camera that does not result from Rayleigh scattering from the beads. When $10 \mu \mathrm{L}$ of the prepared beads solution was pipetted onto the electrode, the scattered light from the beads illuminated the electrode and was refracted toward the microscope objective resulting in a sharp image of the beads without the use of fluorescence. Since the diameter of the beads $(\sim 740 \mathrm{~nm})$ is of the same order as the wavelength of the green LED $(565 \mathrm{~nm})$, the beads appear very bright on a dark background due to Rayleigh scattering. The use of an LED light source with a 45-deg incidence angle, which was simple and cost-effective, precluded the need of a complicated fluorescent enabled sample and fluorescent microscope for the experiments. We then connected the PID electrode array to Tektronix AFG 3021B function generator.

We used a custom-made DEP spectroscopy application software for observation and recording of the results during the experiment. At the beginning, the function generator was automatically set to a positive frequency of $10 \mathrm{kHz}$ to cause positive DEP. Low-frequency electric fields $(<50 \mathrm{kHz})$ induce positive DEP whereas high-frequency electric fields $(>250 \mathrm{kHz})$ induce negative DEP force for the polystyrene beads suspended in the PBS buffer. ${ }^{5}$ The positive DEP force resulted in the attraction of the beads toward the edge of the electrodes. A bright layer produced by the light scattering from the beads can be seen forming at the edge of the electrodes in Fig. 2(a). Once the beads form a layer at the edge of the electrode, our software automatically switched the frequency to one of the frequencies that produces negative DEP force. The negative DEP force produces a strong repulsion force on the beads from the edges of the electrodes. We tracked and recorded the repulsion velocity of the beads through our software and calculated the velocity $(\mu \mathrm{m} / \mathrm{s})$ of the repulsed beads as they travel away from the electrode edge through image processing.

We measured the variation of the light intensity with respect to the horizontal position in the region of interest at different time intervals as shown in Fig. 3. The peak light intensity observed in the image corresponds to the position of the functionalized beads. We process two images to calculate the velocity of repulsion, one captured at positive DEP and the other just after switching to negative DEP. The shift in the center of mass of the light intensity, which is proportional to the local concentration of the polystyrene beads, is calculated for both images and is used to calculate the velocity of the repulsion due to negative DEP. The results for 10 repetitions of the experiment are recorded in Tables 1 and 2. These 10 experiments were conducted with four different $10 \mu \mathrm{L}$ droplets for each of the two cases considered: $0 \%$ and $0.8 \%$ avidin-biotin conjugation. The PID electrode array was washed with deionized water before a new droplet was applied.

We observed a relationship between the velocity of repulsion of the beads and the applied frequency of the electric field. As we switched to higher frequency electric fields from our

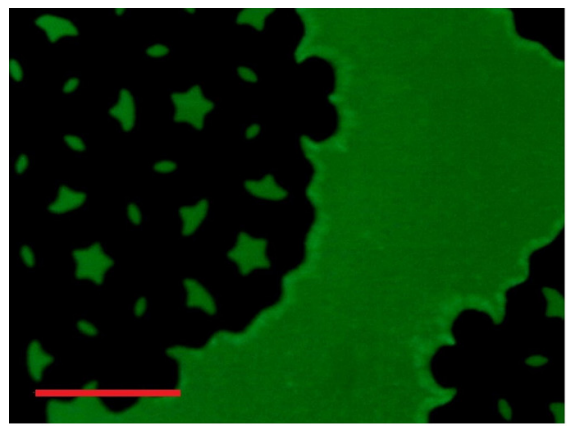

(a)

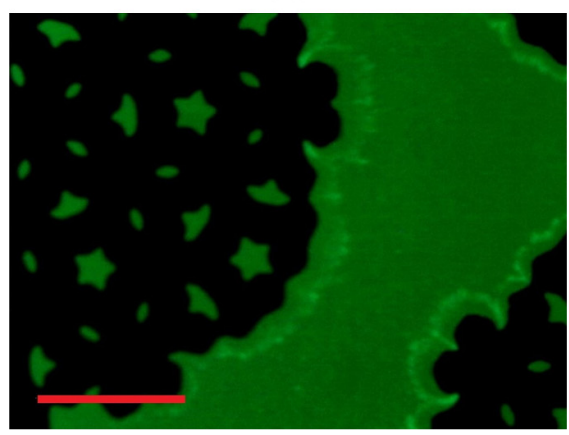

(c)

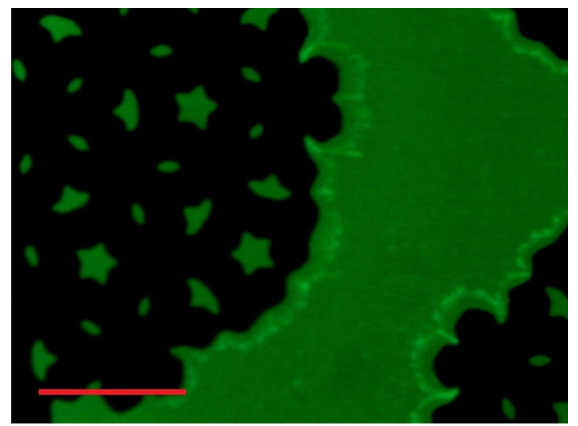

(b)

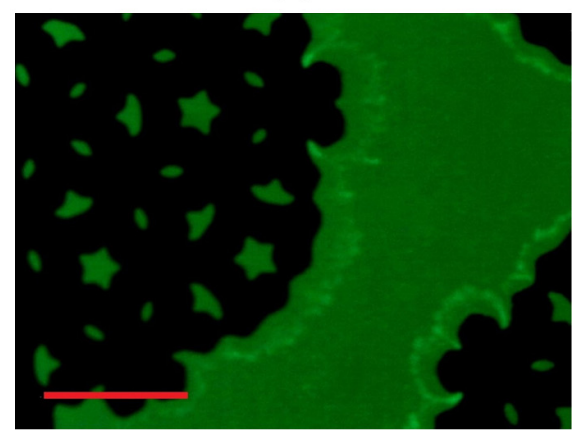

(d)

Fig. 2 Demonstration of negative DEP effect through time-lapse images captured through DEP spectroscopy application (assuming time $=0 \mathrm{~ms}$ when the electric field frequency is changed to induce negative DEP) (a) $t=0 \mathrm{~ms}$, (b) $t=80 \mathrm{~ms}$, (c) $t=160 \mathrm{~ms}$, and (d) $t=240 \mathrm{~ms}$. The electrodes are visible in the darker regions in the picture. The bright layer visible on the edge of the electrode is formed by the accumulation of polystyrene beads. As the frequency is changed to induce negative DEP, the beads are repelled from the electrode. The red scale bar indicates $100 \mu \mathrm{m}$. 


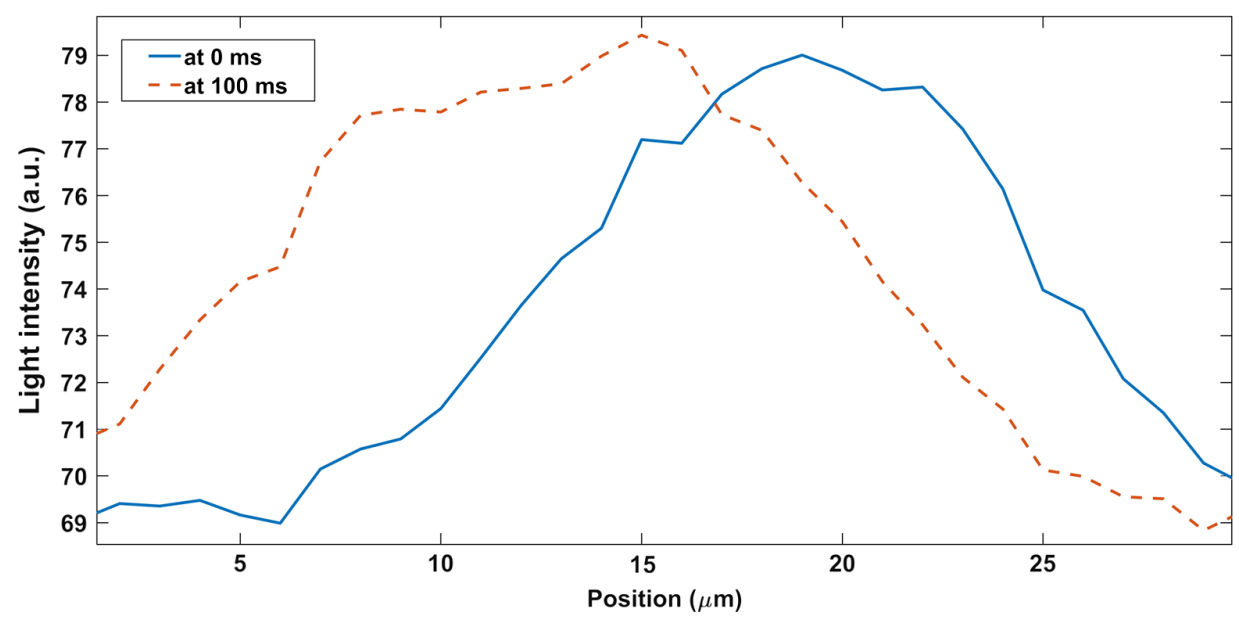

Fig. 3 Light intensity as a function of the distance from the edge of the electrode.

software, the negative DEP force was increased resulting in a higher velocity of repulsion from the edge of the electrode. While switching the frequency, we maintained all other experiment parameters and conditions constant. The results of the negative DEP spectrum obtained in the experiment \#1 shown in Tables 1 and 2 are shown in Fig. 4, along with the standard deviation of a measurement. We observed a significant change in the repulsion velocity of the beads between $0 \%$ avidin-biotin conjugation (i.e., only biotin functionalized polystyrene beads) and $0.8 \%$ conjugation (an average of 80 avidin molecules attached per biotin functionalized polystyrene bead), especially in the frequency range above $1500 \mathrm{kHz}$. This means that our

Table 1 Experiment results with $0 \%$ avidin-biotin binding.

\begin{tabular}{lcccccc}
\hline & \multicolumn{5}{c}{ Frequency $(\mathrm{kHz})$} \\
\cline { 2 - 7 } Experiment & 500 & 800 & 1100 & 1400 & 1700 & 2000 \\
\cline { 2 - 7 } 1 & 58.78 & 63.52 & 63.86 & 66.59 & 67.42 & 68.79 \\
2 & 57.66 & 62.68 & 64.12 & 66.05 & 67.01 & 67.97 \\
3 & 58.35 & 63.49 & 63.64 & 66.19 & 67.79 & 68.96 \\
4 & 57.08 & 63.46 & 64.10 & 66.32 & 67.37 & 68.88 \\
5 & 57.55 & 63.43 & 63.70 & 66.19 & 67.57 & 68.74 \\
6 & 57.47 & 63.55 & 63.68 & 66.17 & 67.63 & 68.78 \\
7 & 57.00 & 63.35 & 63.92 & 66.48 & 67.51 & 68.89 \\
8 & 57.53 & 63.08 & 63.71 & 66.04 & 67.62 & 68.94 \\
9 & 57.23 & 63.12 & 64.06 & 66.50 & 67.39 & 68.72 \\
10 & 57.27 & 63.38 & 63.97 & 66.59 & 67.42 & 68.82 \\
Average & 57.59 & 63.30 & 63.87 & 66.31 & 67.47 & 68.75 \\
Standard deviation & 0.56 & 0.27 & 0.19 & 0.21 & 0.21 & 0.29 \\
\hline & 0.98 & 0.43 & 0.29 & 0.32 & 0.31 & 0.42 \\
\hline
\end{tabular}

experimental setup can detect as little as 80 avidin molecules attached on the biotin functionalized polystyrene bead surface.

The polystyrene beads also experience hydrodynamic drag force due to friction that is opposite in direction to the DEP force. Since the hydrodynamic drag force also depends on the size of the particle, biotin functionalized polystyrene beads with higher concentrations of attached avidin experienced higher drag force. The observed increase in velocity for the beads with $0.8 \%$ of avidin-biotin conjugation when compared to the beads without avidin means that the increase in DEP force was greater than the increase in the hydrodynamic drag force due to the binding of avidin. The graph in Fig. 4 can be considered a calibration

Table 2 Experiment results with $0.8 \%$ avidin-biotin binding.

\begin{tabular}{lllllll}
\hline & \multicolumn{6}{c}{ Frequency $(\mathrm{kHz})$} \\
\cline { 2 - 7 } Experiment & 500 & 800 & 1100 & 1400 & 1700 & 2000 \\
\cline { 2 - 7 } 1 & 62.70 & 65.68 & 72.02 & 80.86 & 84.53 & 88.42 \\
2 & 62.93 & 65.70 & 72.16 & 80.72 & 84.29 & 88.40 \\
3 & 62.57 & 65.48 & 72.53 & 80.75 & 84.35 & 88.39 \\
4 & 62.57 & 65.60 & 72.33 & 80.99 & 84.41 & 88.36 \\
5 & 62.87 & 65.82 & 72.26 & 80.78 & 84.08 & 88.52 \\
6 & 62.75 & 65.68 & 72.17 & 80.45 & 84.39 & 88.57 \\
7 & 62.81 & 65.63 & 72.06 & 80.56 & 84.53 & 88.68 \\
8 & 62.50 & 65.68 & 72.53 & 80.55 & 84.52 & 88.48 \\
9 & 62.69 & 65.87 & 72.44 & 80.59 & 84.23 & 88.43 \\
10 & 62.63 & 65.71 & 72.34 & 80.97 & 84.56 & 88.36 \\
Average & 62.70 & 65.69 & 72.29 & 80.72 & 84.39 & 88.46 \\
Standard deviation & 0.14 & 0.11 & 0.18 & 0.18 & 0.15 & 0.10 \\
\hline & 0.22 & 0.17 & 0.25 & 0.23 & 0.18 & 0.12 \\
\hline
\end{tabular}




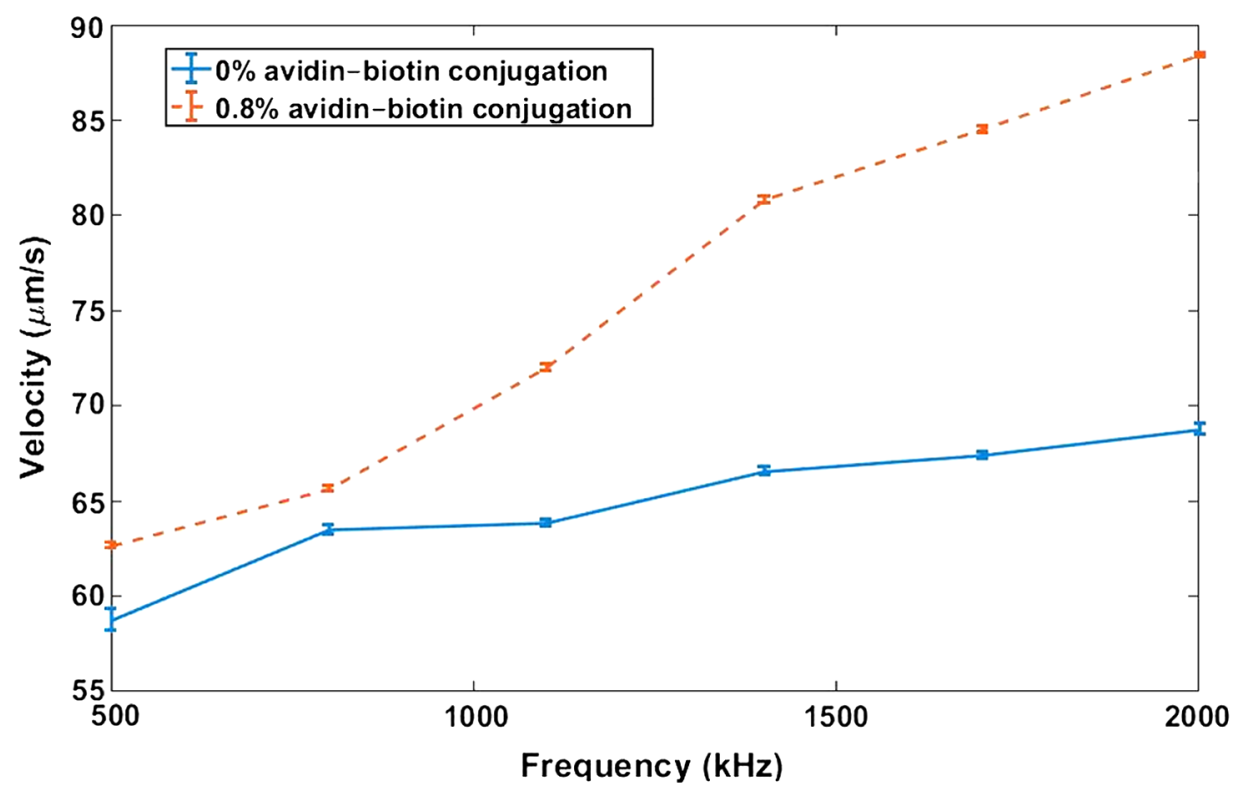

Fig. 4 Negative DEP spectrum of biotin functionalized polystyrene beads with $0 \%$ of $0.8 \%$ of biotin conjugation.

curve for sensing and identification of avidin in biological samples in a medium with the same conductivity.

\section{Conclusion}

We have proposed and validated an automated technique for the detection and quantification of rare analytes in biological samples based on the negative DEP spectroscopy. The early detection and quantification or rare biomolecules can help physicians to save lives from life threatening diseases in early stage, such as cancer and myocardial infarction. We were able to achieve an improvement by 1 order of magnitude in the detection limit of avidin, which we used to demonstrate the technique. Our technique has the potential to be incorporated into a point-ofcare lab-on-a-chip device because of its use of a microscopic electrode array, which can be reutilized after each lab test, and the use of automated software for the detection and quantification of biomolecules that are indicators of diseases. Moreover, the image acquisition system collects the Rayleigh scattering from polystyrene beads illuminated by an LED source with 45-deg incidence. Therefore, there is no need to use fluorescence markers and fluorescence filters in this method. We are extending this work by improving the electrode design and the experimental procedure to improve the spectral resolution of negative DEP spectroscopy to further lower the detection limit of rare biomolecules in biological samples.

\section{Disclosures}

No conflicts of interest, financial or otherwise, are declared by the authors.

\section{References}

1. L. M. Broche et al., "Early detection of oral cancer-is dielectrophoresis the answer?" Oral Oncol. 43(2), 199-203 (2007).

2. J. F. Rusling et al., "Measurement of biomarker proteins for point-ofcare early detection and monitoring of cancer," Analyst 135(10), 2496-2511 (2010).
3. J. S. Daniels and N. Pourmand, "Label-free impedance biosensors: opportunities and challenges," Electroanalysis 19(12), 1239-1257 (2007).

4. R. Pethig and S. Smith, Introductory Bioelectronics: For Engineers and Physical Scientists, Wiley, West Sussex (2012).

5. L. Velmanickam, D. Laudenbach, and D. Nawarathna, "Dielectrophoretic label-free immunoassay for rare-analyte quantification in biological samples," Phys. Rev. E 94(4), 042408 (2016).

6. B. V. Chikkaveeraiah et al., "Electrochemical immunosensors for detection of cancer protein biomarkers," ACS Nano 6(8), 6546-6561 (2012).

7. J. Wu et al., "Biomedical and clinical applications of immunoassays and immunosensors for tumor markers," TrAC Trends Anal. Chem. 26(7), 679-688 (2007).

8. H. Chen et al., "Protein chips and nanomaterials for application in tumor marker immunoassays," Biosens. Bioelectron. 24(12), 3399-3411 (2009).

9. A. A. Panackal et al., "Enzyme immunoassay versus latex agglutination cryptococcal antigen assays in adults with non-HIV-related cryptococcosis," J. Clin. Microbiol. 52(12), 4356-4358 (2014).

10. I. C. Shekarchi et al., "Avidin-biotin latex agglutination assay for detection of antibodies to viral antigens," J. Clin. Microbiol. 26(5), 954-956 (1988).

11. J. A. Molina-Bolívar and F. Galisteo-González, "Latex immunoagglutination assays," J. Macromol. Sci. Part C Polym. Rev. 45(1), 59-98 (2005).

12. F. J. Gella, J. Serra, and J. Gener, "Latex agglutination procedures in immunodiagnosis," Pure Appl. Chem. 63(8), 1131-1134 (1991).

13. H.-H. Chen et al., "High-purity separation of cancer cells by optically induced dielectrophoresis," J. Biomed. Opt. 19(4), 045002 (2014).

14. S. Gupta et al., "On-chip latex agglutination immunoassay readout by electrochemical impedance spectroscopy," Lab Chip 12(21), 42794286 (2012).

15. M. C. Rodriguez, A.-N. Kawde, and J. Wang, "Aptamer biosensor for label-free impedance spectroscopy detection of proteins based on recognition-induced switching of the surface charge," Chem. Commun. (Camb) 287(34), 4267-4269 (2005).

16. M. Varshney et al., "A label-free, microfluidics and interdigitated array microelectrode-based impedance biosensor in combination with nanoparticles immunoseparation for detection of Escherichia coli O157:H7 in food samples," Sens. Actuators B Chem. 128(1), 99-107 (2007).

17. H. Cai, T. M. H. Lee, and I. M. Hsing, "Label-free protein recognition using an aptamer-based impedance measurement assay," Sens. Actuators B Chem. 114(1), 433-437 (2006).

18. Z. Zou et al., "Functionalized nano interdigitated electrodes arrays on polymer with integrated microfluidics for direct bio-affinity sensing 
using impedimetric measurement," Sens. Actuators A Phys. 136(2), 518-526 (2007).

19. R. Pethig, "Dielectrophoresis: status of the theory, technology, and applications," Biomicrofluidics 4(2), 022811 (2010).

20. G. L. Hornyak et al., Fundamentals of Nanotechnology, CRC Press, Boca Raton, Florida (2008).

21. T. B. Jones, "Basic theory of dielectrophoresis and electrorotation," IEEE Eng. Med. Biol. Mag. 22(6), 33-42 (2003).

22. N. R. Shanmugam, S. Muthukumar, and S. Prasad, "Ultrasensitive and low-volume point-of-care diagnostics on flexible strips-a study with cardiac troponin biomarkers," Sci. Rep. 6, 33423 (2016).

23. A. K. Martin et al., "Troponin elevations in patients with chronic cardiovascular disease: an analysis of current evidence and significance," Ann. Card. Anaesth. 19(2), 321-327 (2016).

24. M. A. Daubert and A. Jeremias, "The utility of troponin measurement to detect myocardial infarction: review of the current findings," Vasc. Health Risk Manage. 6(1), 691-699 (2010).

25. L. Velmanickam and K. Nawarathna, "Dielectrophoretic cell isolation in microfluidics channels for high-throughput biomedical applications," in 2016 IEEE Int. Conf. on Electro Information Technology (EIT), pp. 302-306 (2016).

26. E. Papagiakoumou et al., "Evaluation of trapping efficiency of optical tweezers by dielectrophoresis," J. Biomed. Opt. 11(1), 014035 (2006).

27. B. P. Lynch, A. M. Hilton, and G. J. Simpson, "Nanoscale dielectrophoretic spectroscopy of individual immobilized mammalian blood cells," Biophys. J. 91(7), 2678-2686 (2006).

28. A. Castellanos et al., "Electrohydrodynamics and dielectrophoresis in microsystems: scaling laws," J. Phys. D. Appl. Phys. 36(20), 25842597 (2003).

29. P. Y. Weng et al., "Size-dependent dielectrophoretic crossover frequency of spherical particles," Biomicrofluidics 10(1), 011909 (2016).

30. F. Camacho-Alanis and A. Ros, "Protein dielectrophoresis and the link to dielectric properties," Bioanalysis 7(3), 353-371 (2015).

31. B. Yafouz, N. A. Kadri, and F. Ibrahim, "Dielectrophoretic manipulation and separation of microparticles using microarray dot electrodes," Sensors 14(4), 6356-6369 (2014).

32. L. Cui, D. Holmes, and H. Morgan, "The dielectrophoretic levitation and separation of latex beads in microchips," Electrophoresis 22(18), 3893-3901 (2001).
33. N. G. Green and H. Morgan, "Dielectrophoresis of submicrometer latex spheres. 1. Experimental results," J. Phys. Chem. B 103(1), 41-50 (1998).

Syed Abdul Mannan Kirmani is a PhD candidate in the Department of Electrical and Computer Engineering, North Dakota State University (NDSU). He received his MS degree in electrical and communication engineering from Myongji University, South Korea, and his BE degree in electrical engineering from the National University of Sciences and Technology, Pakistan. His research interests include optical fiber communications and biomedical engineering. $\mathrm{He}$ is a member of IEEE-Eta Kappa Nu and Phi Kappa Phi Honor Society.

Fleming Dackson Gudagunti received his BE degree in electrical and electronics engineering from Visvesvaraya Technological University, India, in 2009 and his MTech degree in VLSI and embedded systems from the Visvesvaraya Technological University, India, in 2014. $\mathrm{He}$ is a PhD student at NDSU, Fargo. His research interests include biomedical engineering and biophotonics.

Logeeshan Velmanickam is a $\mathrm{PhD}$ candidate in the Department of Electrical and Computer Engineering with biomedical emphasis at NDSU. He received his BS degree in electrical and electronic engineering from the University of Peradeniya, Sri Lanka, in 2015. His research focuses on developing biosensing techniques that are utilized in the detection of rare target molecules in biological samples.

Dharmakeerthi Nawarathna received his PhD in 2005. Currently, he is an assistant professor in the Department of Electrical and Computer Engineering, NDSU. His current research focuses on developing labon-a-chip techniques for biomedical applications.

Ivan T. Lima, Jr. received his $\mathrm{PhD}$ in electrical engineering from the University of Maryland, Baltimore County, in 2003. In 2003, he became a faculty member in the Department of Electrical and Computer Engineering, NDSU, where he is currently an associate professor with tenure. His research interests include optical communications and biomedical engineering. He is a member of SPIE, OSA, and a senior member of the IEEE Photonics Society. 